Review Article

\title{
Carbapenemase-Producing Non-Glucose-Fermenting Gram-Negative Bacilli in Africa, Pseudomonas aeruginosa and Acinetobacter baumannii: A Systematic Review and Meta-Analysis
}

\author{
Mizan Kindu $\mathbb{D}^{\text {, }},{ }^{1}$ Lemma Derseh, ${ }^{2}$ Baye Gelaw, ${ }^{1}$ and Feleke Moges ${ }^{1}$ \\ ${ }^{1}$ Department of Medical Microbiology, College of Medicine and Health Science, University of Gondar, Gondar, \\ P.O. Box 196, Ethiopia \\ ${ }^{2}$ Department of Epidemiology and Biostatistics Institute of Public Health College of Medicine and Health Sciences, \\ University of Gondar, Gondar, P.O. Box 196, Ethiopia
}

Correspondence should be addressed to Mizan Kindu; mizankindu00@gmail.com

Received 7 February 2020; Accepted 20 October 2020; Published 4 November 2020

Academic Editor: Simona Nardoni

Copyright (c) 2020 Mizan Kindu et al. This is an open access article distributed under the Creative Commons Attribution License, which permits unrestricted use, distribution, and reproduction in any medium, provided the original work is properly cited.

\begin{abstract}
Background. Studies have reported that the existence of CP bacteria in Africa, but, in general, comprehensive data about the molecular epidemiology of CP organisms are limited. Therefore, this systematic review and meta-analysis expound the pooled prevalence of CP P. aeruginosa and CP A. baumannii clinical isolates in Africa. It also identified the diversity of carbapenemases or their encoding genes among the isolates in Africa. Lastly, the review observed the trends of these CP isolates in Africa. Methods. A comprehensive search was performed between July 2019 and October 2019 in the following databases: PubMed, Google Scholar, and African Journal online. The included articles were published only in English. The screening was done by two authors independently. The data extracted on Excel spreadsheet were transferred to STATA 11 software for analysis. Results. From a total of 1,454 articles searched, 42 articles were eligible. Most of the studies were conducted in the North Africa region. But there was no report from Central Africa. The pooled prevalence of CP P. aeruginosa and CP A. baumannii among the clinical specimens in Africa was $21.36 \%$ and $56.97 \%$, respectively. OXA-23 and VIM were the most prevailing carbapenemase among P. aeruginosa and A. baumannii, respectively. The cumulative meta-analysis revealed a relative increment of the prevalence of CP P. aeruginosa over time in Africa but it showed a higher prevalence of CP A. baumannii isolates across years. Conclusion. The review revealed a high pooled prevalence of CP A. baumannii clinical isolates in Africa which needs urgent action. Moreover, the emergence of concomitant carbapenemases, especially OXA-23 + NDM among CP A. baumannii, was also an alarming problem.
\end{abstract}

\section{Introduction}

Carbapenem-resistant (CR) Acinetobacter baumannii (CRAB) and CR Pseudomonas aeruginosa (CRPA) are among the top tier of the World Health Organization (WHO) list of antibiotic-resistant "priority pathogens" that pose the greatest threat to human health [1]. Infections with these resistant bacteria are a matter of national and international concern as they are an emerging cause of Hospital Acquired Infections (HAIs) that pose a significant threat to public health and responsible for hospital outbreaks worldwide [2]. Moreover, they are associated with high rates of morbidity and mortality, especially in patients with serious underlying disorders or patients admitted to the intensive care unit (ICU) [3].

Although carbapenem antibiotics were introduced to treat infection caused by bacteria resistant to penicillin, cephalosporins, and fluoroquinolones, the reliability of these antibiotics has been reduced because of resistance. Multiple mechanisms of carbapenem resistance have been identified 
including overexpression of efflux pumps, porin mutations, and enzymatic inactivation [4].

The production of an enzyme carbapenemase, having the ability to hydrolyze almost all beta-lactam antibiotics, has been the most critical mechanism that causes resistance to carbapenems [5]. Carbapenemases belong to classes A, B, and $\mathrm{D}$ of ambler classification beta-lactamases [6]. Carbapenemase that belongs to class A beta-lactamase utilizes serine for $\beta$-lactam hydrolysis that contains a wide variety of enzymes including KPC (Klebsiella pneumoniae carbapenemase $=$ KPC-2 to KPC-13), IMI (Imipenem-hydrolyzing $\beta$-lactamase; IMI-1 to IMI- 3), and GES (Guiana extended spectrum; GES-1 to GES-20), with hydrolyzing activity against carbapenems. These enzymes are inhibited by clavulanic acid. The KPCs are the most frequently identified class A carbapenemases [5].

Likewise, these belong to class $\mathrm{D}$ beta-lactamases (oxacillinase $=$ OXA-types) and also utilize the serine-active site for $\beta$-lactam hydrolysis. OXA enzymes (the name came since they hydrolyze isoxazolyl penicillins much faster than penicillins) were recently divided into 12 subgroups: OXA-23-like, OXA24/40-like, OXA-48, OXA-51-like, OXA-58-like, OXA-143-like, OXA-253, OXA-211, OXA-213, OXA- 214, OXA-229, and OXA-235 [7]. The OXA-23, OXA-24/40, OXA-48, and OXA-58 carbapenemases, which are mainly plasmid-encoded, and the OXA-51 carbapenemase, which is chromosomally encoded and intrinsic (naturally found) in A. baumannii. Class D enzymes are not inhibited by clavulanic acid or ethylenediaminetetraacetic acid (EDTA) [8].

On the contrary, carbapenemases found under class B beta-lactamases (also referred metallo-beta-lactamase $=\mathrm{MBL})$ use active-site zinc to hydrolase all $\beta$-lactams except for aztreonam, a monobactam, and are susceptible to inhibition by EDTA. These enzymes include but are not limited to VIM (Verona integron-encoded metallo-betalactamase), GIM (German imipenemase), IMP, AIM-1 (Adelaide imipenemase 1), SIM (Seoul imipenemase), and NDM (New Delhi metallo-beta-lactamase) [9].

Studies have reported that the existence of carbapenemase-producing (CP) bacteria in Africa [10-13] but in general comprehensive data about the molecular epidemiology of CP organisms in Africa is limited. Therefore, this systematic review and meta-analysis expound the pooled prevalence of CPPA and CPAB clinical isolates in Africa. Moreover, it also systematically reviews the diversity of carbapenemases and their encoding genes among these $\mathrm{CP}$ clinical isolates in Africa. Lastly, the review observed the trends of these isolates in Africa.

\section{Methods}

2.1. Protocol Registration and Review Reporting. This systematic review and meta-analysis was registered at the international prospective register of systematic review and meta-analysis (PROSPERO) with the registration number of CRD42019147430. The results of this review were reported based on the Preferred Reporting Items for Systematic Review and Meta-Analysis (PRISMA) statement guideline [14].
2.2. Searching Strategy and Information Sources. To find potentially relevant articles, comprehensive searches were performed between July 2019 and October 2019 in the following databases: PubMed/MEDLINE, Google Scholar, and African Journal online. All searches were limited to articles written in English. The search was also supplemented by searching gray literature of observational study and reference lists of the eligible studies.

The search strings or terms were stemmed from the isolates of each individual. For $P$. aeruginosa, we used $P$. aeruginosa, carbapenemase-producing $P$. aeruginosa, class A carbapenemases, class B carbapenemases, Metallo$\beta$-lactamase, class $\mathrm{D}$ carbapenemases, oxacillinase, and Africa. For A. baumannii, we used A. baumannii, carbapenemase-producing $A$. baumannii, class A carbapenemases, class B carbapenemases, Metallo- $\beta$-lactamase, class D carbapenemases, oxacillinase, and Africa. In the advanced searching databases, the searching strategy was built based on the abovementioned terms using the "Medical Subject Headings (MeSH)" and "All fields" by linking "AND" and "OR" Boolean operator terms as appropriate.

2.3. Inclusion and Exclusion Criteria. We included studies reporting the prevalence of $\mathrm{CPPA}$ and $\mathrm{CPAB}$ infection among clinical specimens recovered from human patients, cross-sectional studies, studies published in English, and studies that detected carbapenemase producers using phenotypic and confirmed through molecular methods.

Studies were excluded if they reported carriage rather than infection, conducted environments, and detected carbapenemase production only using the phenotypic method. Review articles, meta-analyses, case reports, case series, letters to the editors, correspondence, outbreak settings, and articles which were not fully accessible after at least two-email contact with the corresponding author were also excluded. The exclusion of these articles was because of the inability to assess the quality of articles in the absence of full text.

2.4. Quality Assessment. Three independent reviewers (MK, $\mathrm{BG}$, and FM) examined the quality of the included studies. Since all the included studies were cross-sectional in nature, the quality of each article was assessed using the Joanna Briggs Institute (JBI) critical appraisal tool prepared for cross-sectional studies [15]. The quality scales of primary studies were considered as low risk for both systematic review and meta-analysis if the studies got $50 \%$ and above.

2.5. Data Extraction Process. Two reviewers (MK and BG) independently extracted data including the name of the first author, year of publication and sample collection, the study design, the total number of each bacterial isolates identified, the number of $\mathrm{CP}$ isolates, the number and type of the carbapenemase enzyme or encoding genes detected for each specific bacterial isolate, the number of CR isolates from antibiotic resistance susceptibility test result, and countries in which the study was done. When disagreement occurred 


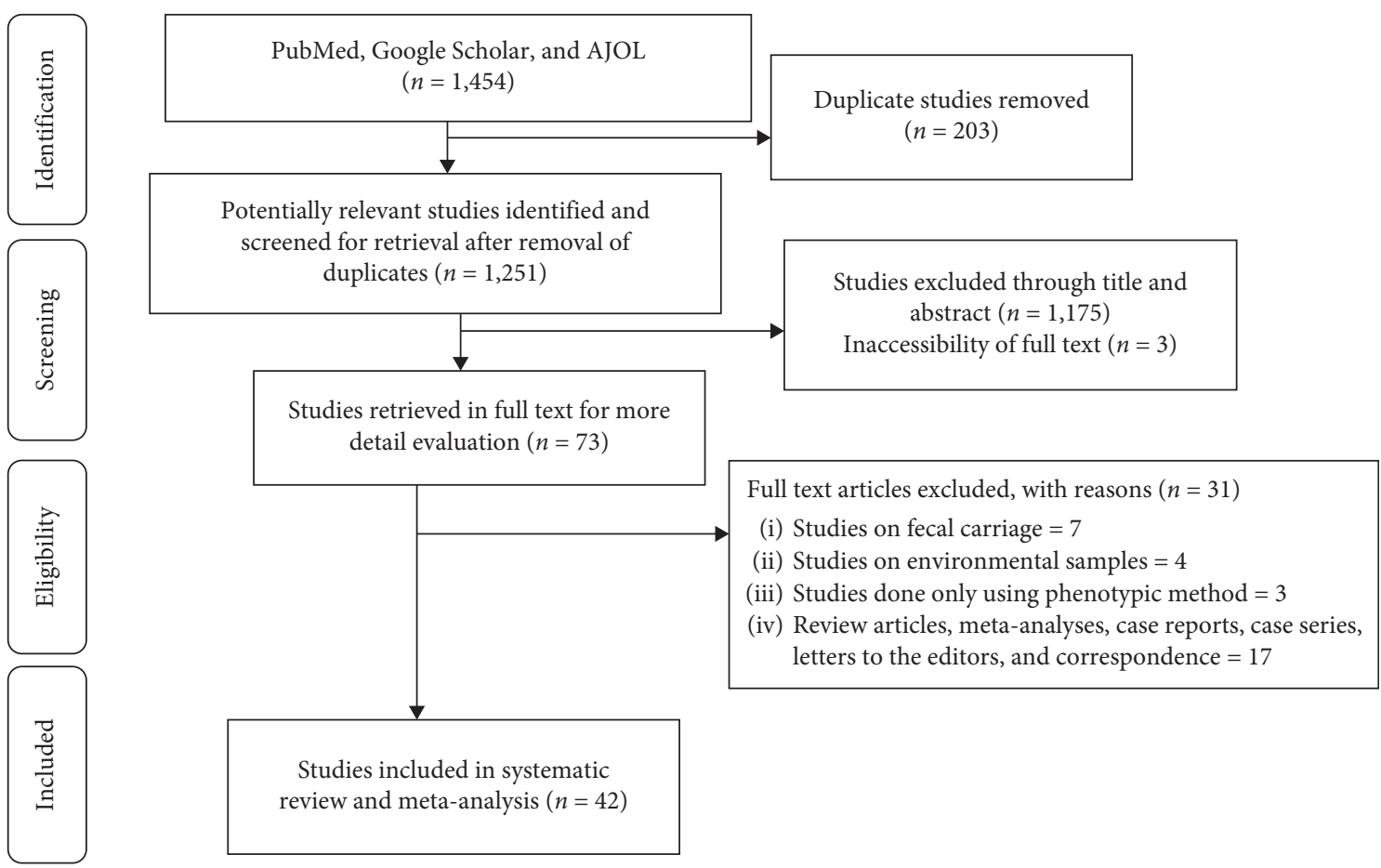

FIgURE 1: Flow chart shows the study selection process.

in the process of data extraction, it was resolved by discussion and consensus. The third and the fourth authors (FM and LG) were consulted when consensus could not be achieved.

2.6. Outcome Measures. The primary outcome measure was the prevalence of CP P. aeruginosa and A. baumannii isolates. The prevalence of carbapenemase producers for each of the isolates was calculated independently as follows. The first was by dividing the total number of $\mathrm{CP}$ isolates by the total number of the identified isolates. The second was calculated by dividing the total number of $\mathrm{CP}$ isolates by their total number of bacterial isolates resistance to carbapenem through antibiotic susceptibility testing. The secondary outcome was the identification of the detected carbapenemase enzymes or their encoding genes for each specific bacterial isolates. The last outcome was also the time trend analysis for the prevalence of $\mathrm{CP}$ isolates in Africa.

2.7. Data Processing and Analysis. The data extracted in Microsoft Excel format was analyzed using STATA Version 11 statistical software. The existence of heterogeneity among studies was examined by the forest plot as well as the $I^{2}$ heterogeneity test, in which $0-40 \%, 50-60 \%, 50-90 \%$, and 75-100\% represented low, moderate, substantial, and considerable heterogeneity, respectively $[16,17]$. The $I^{2}$ heterogeneity test $\geq 50 \%$ and a $p$ value of $<0.05$ assured the presence of heterogeneity. Thus, the DerSimonian-Laird random-effects model was employed [18]. To identify the influential studies that resulted in variation, a sensitivity analysis was carried out using the "metaninf" command [19]. Similarly, subgroup analyses were also employed by assuming the region as grouping variables and sources of variation.

Furthermore, using the "metafunnel" command [20] and objectively by Egger's regression test, publication bias was detected [20]. Accordingly, asymmetry of the funnel plot and/or statistical significance of Egger's regression test $(p$ value $<0.05$ ) was suggestive of publication bias [21]. Finally, all statistical interpretations were reported based on the $95 \% \mathrm{CI}$.

\section{Results}

3.1. Characteristics of the Included Studies. During our literature search, 1,454 potentially relevant articles were identified, of which 203 duplicated articles were excluded (Figure 1). All titles and abstracts of the remaining 1,251 articles were screened, resulting in the exclusion of 1,175 publications. Moreover, 3 articles were also excluded due to the inaccessibility of full text. The remaining 73 articles underwent a second screening based on the inclusion and exclusion criteria of the review and 31 of them were excluded. Finally, 42 articles were included in the systematic review and meta-analysis (Figure 1).

Among the articles, those which fulfill eligibility criteria of this systematic review and meta-analysis, most of the studies reporting molecular identification of CPPA and $\mathrm{CPAB}$ were conducted in the North Africa region $(n=32$; $76.19 \%)$. In this region, mainly Egypt $(n=17)$ conducted the detection CP bacterial isolates using molecular methods followed by Algeria $(n=8)$, Morocco $(n=2$, Tunisia $(n=2)$, Sudan $(n=2)$, and Libya $(n=1)$. The remaining 10 studies $(23.81 \%)$ were carried out in other parts of African regions such as Ethiopia $(n=1)$, Uganda $(n=2)$, Tanzania 


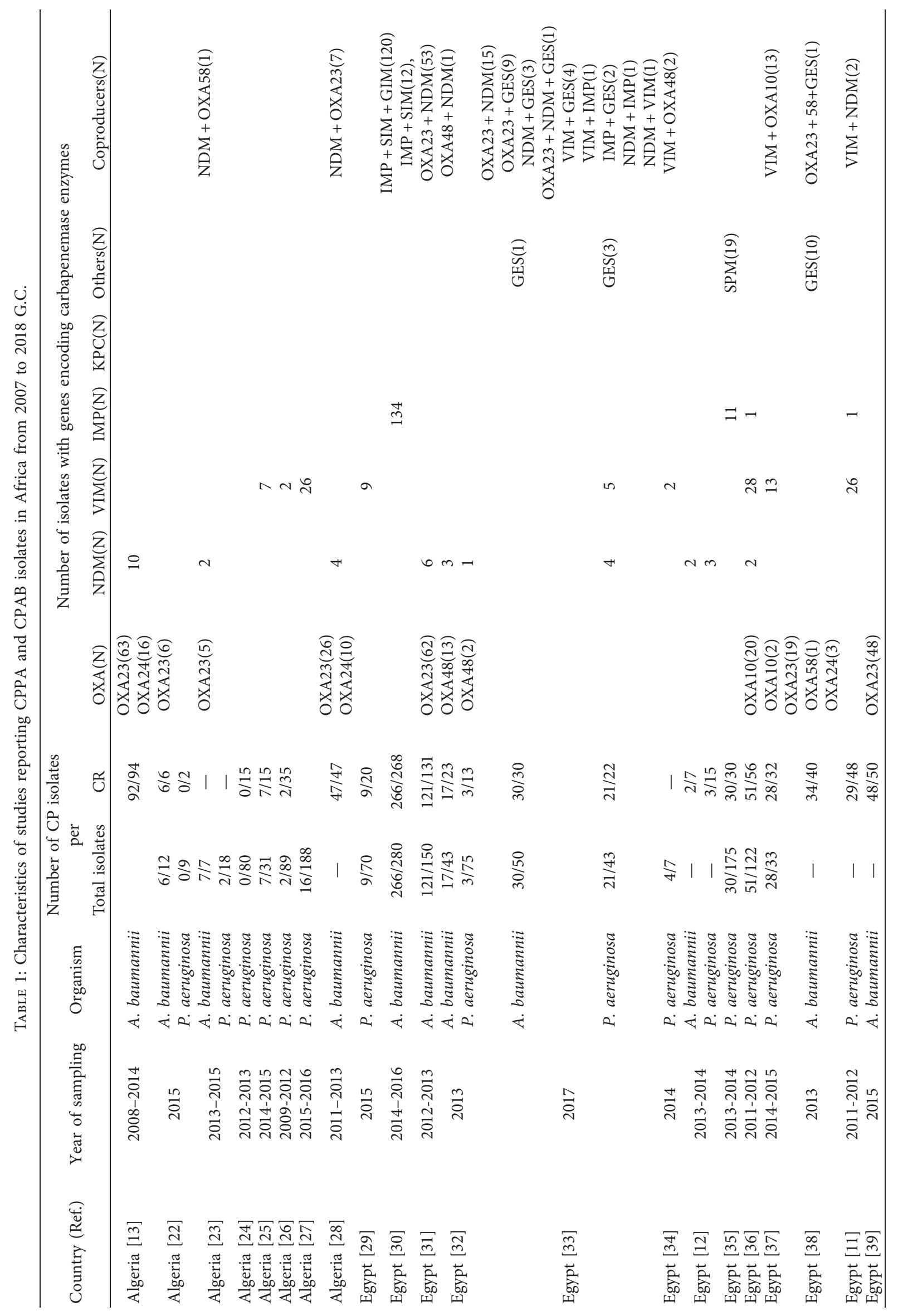




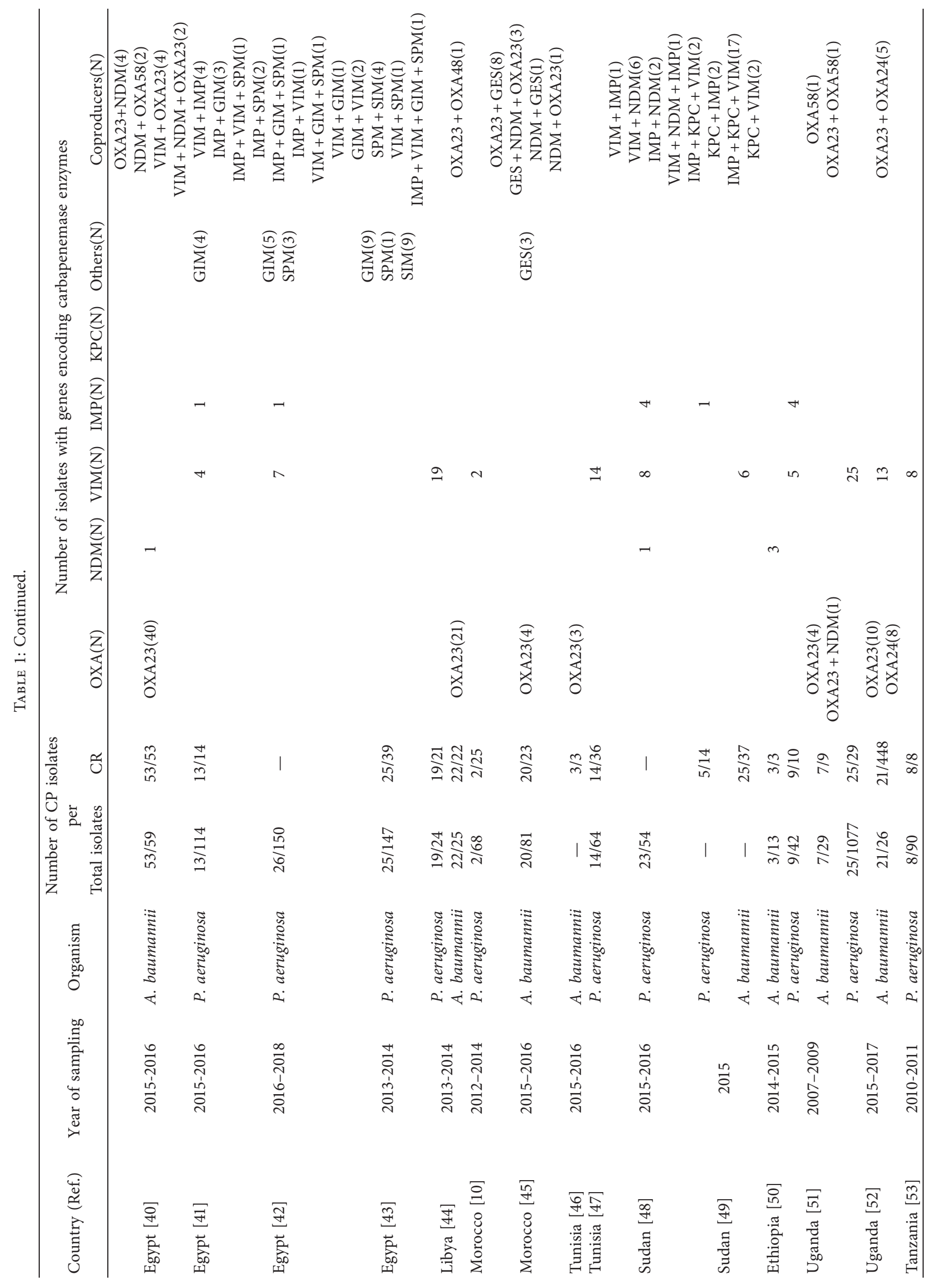




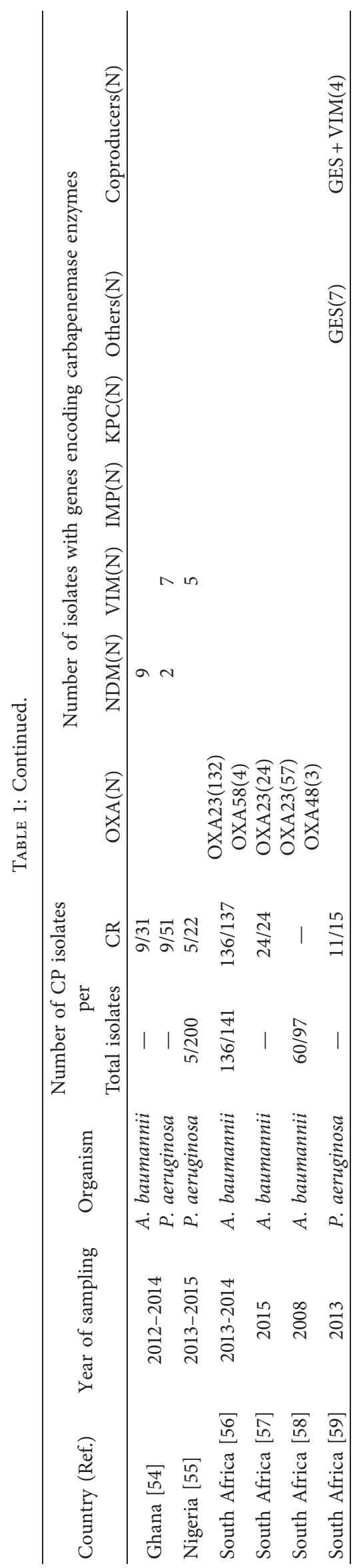




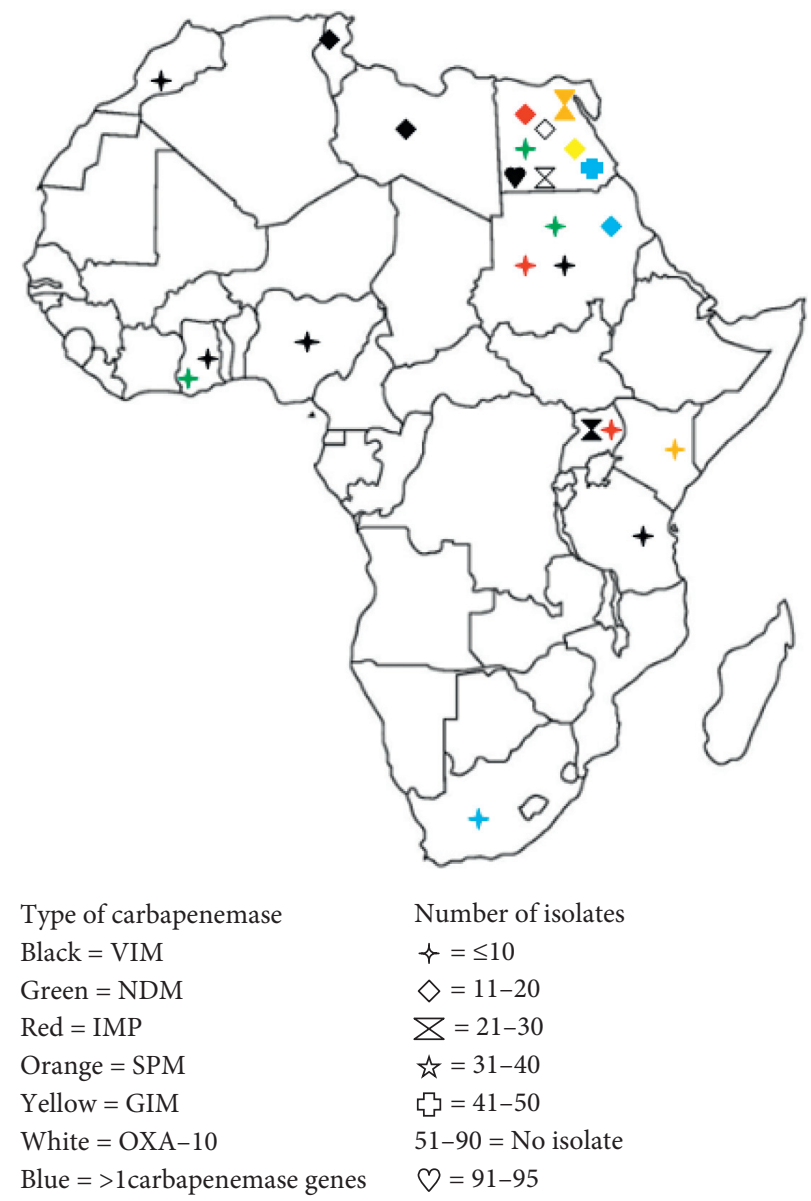

Figure 2: Geographical distribution CPPA isolates in Africa from 2007 to 2018 G.C.

$(n=1)$, Ghana $(n=1)$, Nigeria $(n=1)$, and South Africa $(n=4)$ (Table 1$)$. However, there was no study from Central Africa.

In this systematic review and meta-analysis, clinical samples were collected from 2007 to 2018 G.C. from the abovementioned African countries. Moreover, some of the studies were conducted by focusing only on a single isolate either on " $A$. baumannii" $(n=13)$ or on " $P$. aeruginosa" $(n=18)$. The remaining $(n=11)$ involved multiple Gramnegative bacterial isolates (Table 1$)$.

\subsection{Carbapenemase-Producing P. aeruginosa in Africa}

3.2.1. Study Characteristics. According to the eligibility criteria of this systematic review and meta-analysis, a total of thirty studies reporting CPPA were included. Most of the studies $(n=24)$ reporting CPPA isolates were conducted in North Africa, majorly from Egypt followed by Algeria. The remaining six were carried out in other parts of the African region such as Uganda, Tanzania, Ghana, Nigeria, and South Africa. There was no report from Central Africa (Table 1).

\subsubsection{Geographical Distribution of Carbapenemase-Produc-} ing $P$. aeruginosa in Africa. The prevalence of CPPA among total isolates identified among all studies in North Africa ranged from $0 \%(0 / 9$ [22], $0 / 80$ [24]) to $84 \%(28 / 33)$ [37] while it ranged from $2.3 \%(25 / 1077)$ [52] to $21.4 \%$ (9/42) [51] in East Africa. Among carbapenemase enzymes, VIM was highly detected along with CPPA isolates in the reported African countries. OXA-10, IMP, SPM, NDM, GES, and GIM carbapenemases were also detected among the isolates (Figure 2). As indicated in Figure 2, all of the identified carbapenemase types among $P$. aeruginosa isolates were detected in Egypt. VIM-producing $P$. aeruginosa isolates were also observed in the majority of African countries (Figure 2). A total of 66 CPPA isolates were carbapenemase coproducers, carrying genes encoding more than one carbapenemase (Table 1).

3.2.3. Pooled Prevalence of Carbapenemase-Producing $P$. aeruginosa in Africa. From a total of 2,973 P. aeruginosa isolates collected from patients between 2007 and 2018 in Africa, the pooled prevalence of CPPA was $21.36 \%$. As shown in Figure 3, there is a statistically significant considerable heterogeneity with $\mathrm{I}^{2}=96 \%$ and $p$ value of less than 0.001 . The pooled result indicates further applying of subgroup and sensitivity analysis for detecting the most influential studies and identifying the source of heterogeneity, respectively. Moreover, among CRPA isolates, the pooled prevalence of CPPA was $53.21 \%$ $(I=97.0 \%, p<0.001)$ (Figure S1) in Supplementary Materials. 


\begin{tabular}{|c|c|c|c|}
\hline $\begin{array}{l}\text { Study } \\
\text { ID }\end{array}$ & & ES $(95 \%$ CI) & $\begin{array}{c}\% \\
\text { weight }\end{array}$ \\
\hline Algeria (2016) & $\bullet \quad 1$ & $11.11(-3.41,25.63)$ & 3.75 \\
\hline Algeria (2016) & $\stackrel{1}{\longrightarrow}$ & $22.58(7.86,37.30)$ & 3.72 \\
\hline Algeria (2014) & $\rightarrow \quad 1$ & $2.25(-0.83,5.33)$ & 5.39 \\
\hline Algeria (2019) & 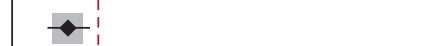 & $13.83(8.90,18.76)$ & 5.22 \\
\hline Egypt (2018) & 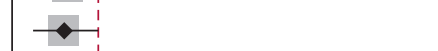 & $12.86(5.02,20.70)$ & 4.84 \\
\hline Egypt (2017) & $\bullet$ & $4.00(-0.43,8.43)$ & 5.27 \\
\hline Egypt (2018) & 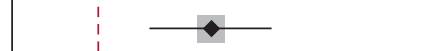 & $48.84(33.90,63.78)$ & 3.69 \\
\hline Egypt (2017) & 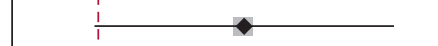 & $57.14(20.48,93.80)$ & 1.39 \\
\hline Egypt (2017) & -1 & $17.14(11.56,22.73)$ & 5.15 \\
\hline Egypt (2014) & $\multimap$ & $41.80(33.05,50.56)$ & 4.70 \\
\hline Egypt (2018) & $\longrightarrow$ & $84.85(72.62,97.08)$ & 4.14 \\
\hline Egypt (2019) & $-\rightarrow$ & $11.40(5.57,17.24)$ & 5.12 \\
\hline Egypt (2019) & -1 & $17.33(11.28,23.39)$ & 5.09 \\
\hline Egypt (2014) & $\rightarrow$ & $17.01(10.93,23.08)$ & 5.09 \\
\hline Libya (2015) & $\longrightarrow$ & $79.17(62.92,95.41)$ & 3.48 \\
\hline Morocco (2016) & $\leftrightarrow \quad$ i & $2.94(-1.07,6.96)$ & 5.31 \\
\hline Tunisia (2019) & $\rightarrow$ & $20.90(11.16,30.63)$ & 4.55 \\
\hline Sudan (2018) & $1 \longrightarrow$ & $42.59(29.40,55.78)$ & 3.97 \\
\hline Uganda (2016) & 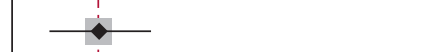 & $21.43(9.02,33.84)$ & 4.11 \\
\hline Uganda (2019) & $\begin{array}{l}1 \\
-\quad 1\end{array}$ & $2.32(1.42,3.22)$ & 5.49 \\
\hline Tanzania (2015) & 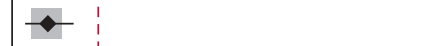 & $8.89(3.01,14.77)$ & 5.11 \\
\hline Nigeria (2018) & 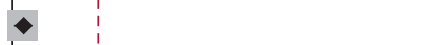 & $2.50(0.34,4.66)$ & 5.44 \\
\hline Algeria (2018) & & (Excluded) & 0.00 \\
\hline Algeria (2015) & 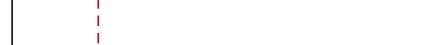 & (Excluded) & 0.00 \\
\hline Overall $(I$-squared $=96.0 \%, p=0.000)$ & \langle & $21.36(16.37,26.36)$ & 100.00 \\
\hline NOTE: weights are from random-effects analysis & i & & \\
\hline \begin{tabular}{l|l}
+ &
\end{tabular} & \\
\hline-97.1 & \multicolumn{3}{|l|}{$\begin{array}{c}1 \\
97.1\end{array}$} \\
\hline
\end{tabular}

FIgURE 3: Forest plot for the pooled prevalence of CPPA isolates in Africa from 2007 to 2018 G.C.

3.2.4. Subgroup Analysis. The results of the subgroup analysis done by considering both regions (Figure S2) in Supplementary Materials and country variables showed that there was still considerable heterogeneity (Figure 4).

3.2.5. Sensitivity Analysis. The result of the sensitivity analysis showed that there was no outlier study, which had an impact on the overall estimation (Figure 5).

3.2.6. Publication Bias. A funnel plot showed a symmetrical distribution (Figure 6). Egger's regression test $p$ value was also 0.909 , which indicated no evidence for publication bias (Table S3) in Supplementary Materials.

3.2.7. Time Trend Analysis of Carbapenemase-Producing $P$. aeruginosa in Africa. The cumulative meta-analysis result showed a relative increment trend of CPPA isolates over time in Africa among the studies published from 2014 to 2018 (Figure 7).

\subsection{Carbapenemase-Producing A. baumannii in Africa}

3.3.1. Study Characteristics. A total of 23 studies that fulfilled the inclusion criteria of this systematic review and meta-analysis reporting $\mathrm{CPAB}$ were involved. Except for seven studies that were conducted in Uganda (2/23), Ethiopia (1/23), Ghana (1/23), and South Africa (3/23), the rest of the studies $(n=16)$ were done in North Africa. The majority of studies reporting $\mathrm{CPAB}$ in North Africa were from Egypt (8/16) followed by Algeria (4/16) (Table 1).

3.3.2. Geographical Distribution of Carbapenemase-Producing A. baumannii in Africa. The lowest prevalence of $\mathrm{CPAB}$ among the total isolates identified in Africa was 4.7\% (21/ 448), reported from Uganda [52], whereas the higher prevalence was $100 \%$ (7/7), reported from a study conducted in Algeria [23]. As shown in Figure 8, the most prevailing carbapenemase enzymes produced by A. baumannii isolates were OXA-23. Likewise, other carbapenemases such as IMP, NDM, and OXA-24 were also detected. But OXA-48, OXA-58, and GES carbapenemases 


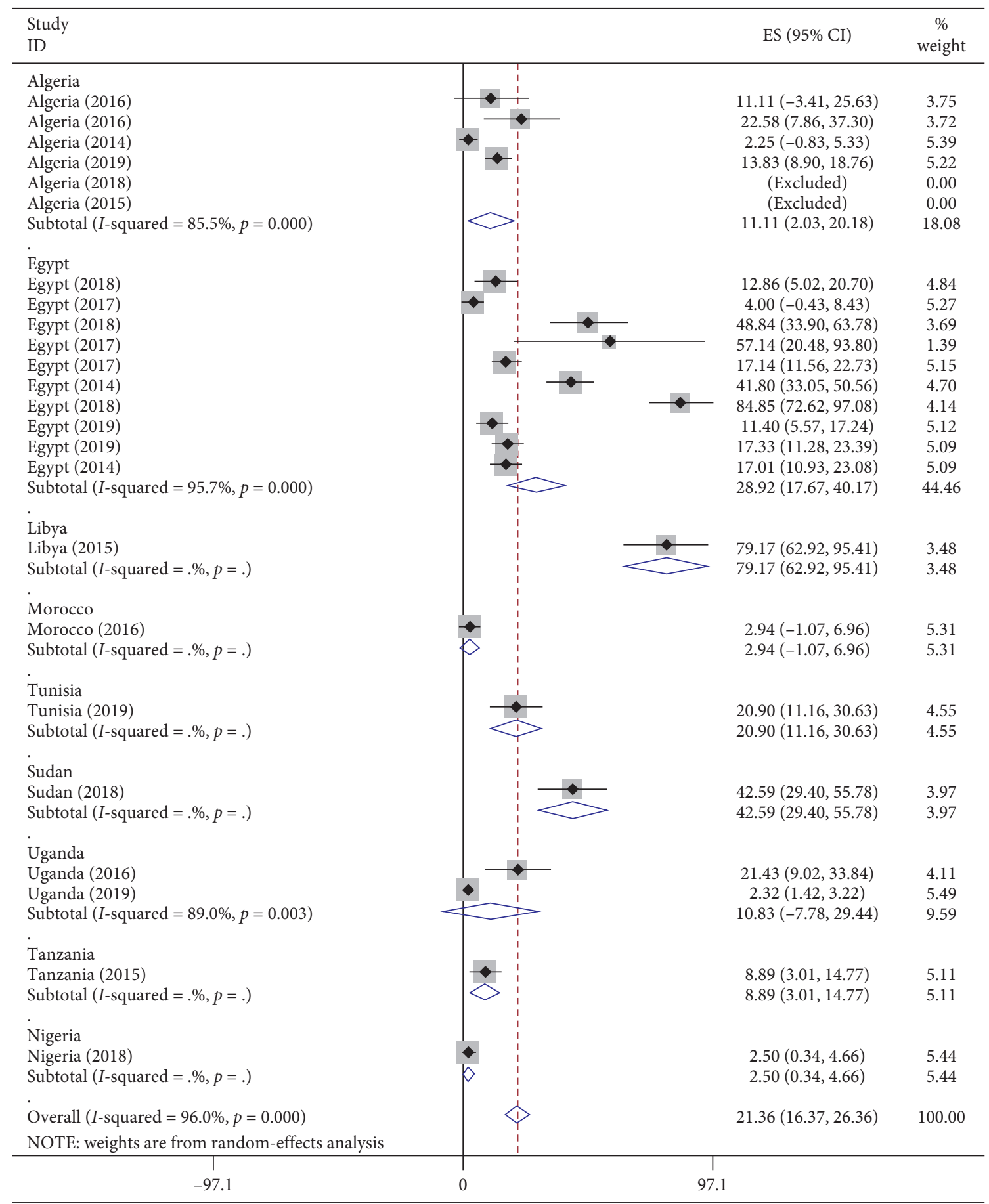

FIGURE 4: Subgroup analysis for the pooled prevalence of CPPA isolates in Africa by country from 2007 to 2018 G.C.

were reported rarely (Table 1). Three or more types of carbapenemases were reported from Algeria, Egypt, Libya, and Uganda (Figure 8). Likewise, a high number of OXA23-producing A. baumannii isolates were reported from Algeria, Egypt, and South Africa. Moreover, OXA-23 carbapenemases had also wider distribution along the studied African countries (Figure 8). The number of A. baumannii clinical isolates that coproduced more than one carbapenemase type was 273. Moreover, $\mathrm{CPAB}$ isolates coproducing OXA-23 + NDM had wider distributions (Table 1).

3.3.3. Pooled Prevalence of Carbapenemase-Producing A. baumannii in Africa. Among the total of 1,435 A. baumannii isolates collected from 2007 to 2017 in Africa, 


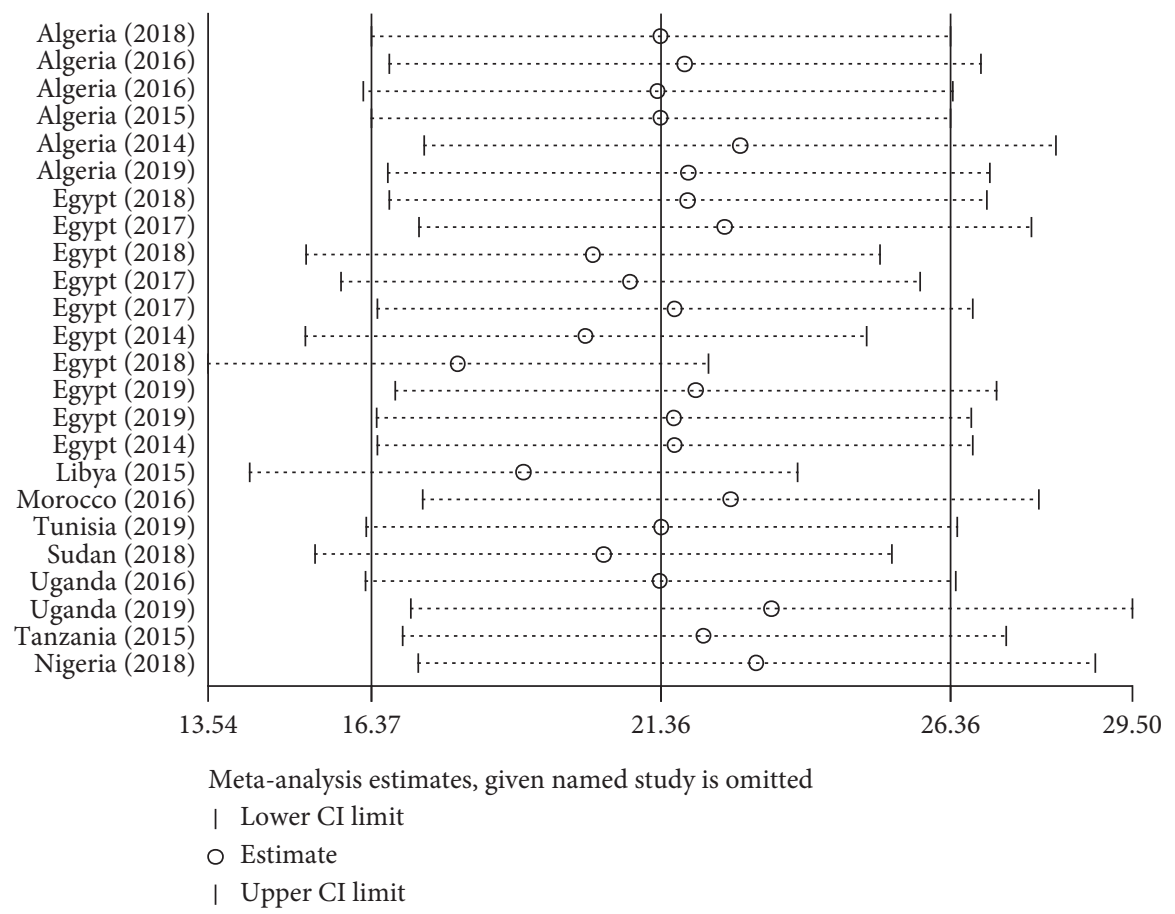

FIgURe 5: Sensitivity analysis for the pooled prevalence of CPPA in Africa from 2007 to 2018 G.C.

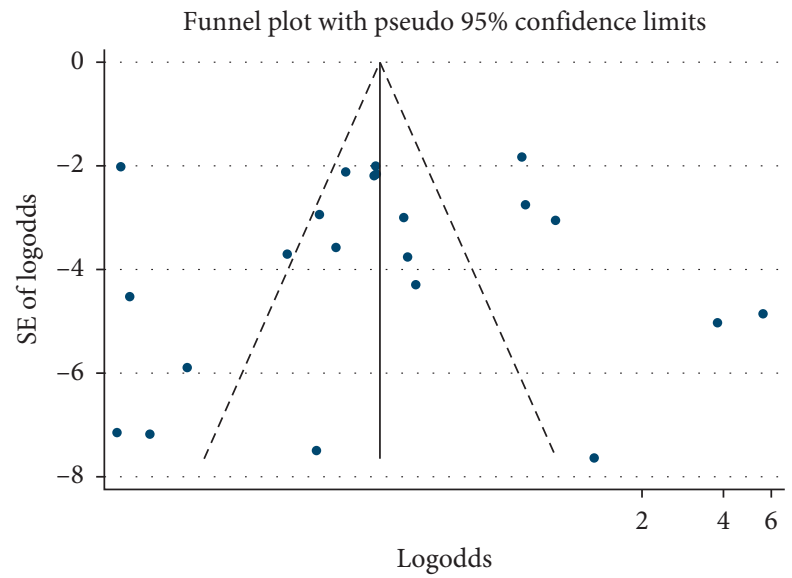

FIgURE 6: Funnel plot for publication bias of pooled prevalence of CPPA in Africa from 2007 to 2018 G.C.

the pooled prevalence of carbapenemase producers was $56.97 \%$ with $\mathrm{I}^{2}$ 99.7\% and $p$ value of less than 0.001 (Figure 9). The pooled prevalence of $\mathrm{CPAB}$ isolate among $\mathrm{CRAB}$ isolates was also $86.11 \%\left(I^{2}=92.4 \%, p<0.001\right)$ (Figure $\left.S 4\right)$ in Supplementary Materials.

3.3.4. Subgroup Analysis. The results of the subgroup analysis done by considering both regions (Figure S5) in Supplementary Materials and country variables (Figure 10) showed still that heterogeneity was considerable.

3.3.5. Sensitivity Analysis. As shown in Figure 11, the prevalence of $\mathrm{CPAB}$ isolates from each study was within the confidence interval limit. This shows that they do not have any influence on the overall estimation.
3.3.6. Publication Bias. In the observational test for publication bias, the funnel plot showed a symmetrical distribution (Figure 12). Likewise, Egger's regression test $p$ value also indicated the absence of publication bias $(p=0.59)$ (Table S6) in Supplementary Materials.

3.3.7. Time Trend Analysis of Carbapenemase-Producing A. baumannii in Africa. According to the cumulative metaanalysis of papers published from 2015 to 2018, CPAB clinical isolates showed high prevalence across the years in the studied African countries (Figure 13).

\section{Discussion}

This systematic review and meta-analysis showed the pooled prevalence of $\mathrm{CPPA}$ and $\mathrm{CPAB}$ among the clinical 


\begin{tabular}{|c|c|c|}
\hline $\begin{array}{l}\text { Study } \\
\text { ID }\end{array}$ & & ES $(95 \%$ CI $)$ \\
\hline Algeria (2014) & - & $2.25(1.94,2.56)$ \\
\hline Egypt (2014) & $\longrightarrow$ & $22.02(-16.74,60.79)$ \\
\hline Egypt (2014) & $\longrightarrow$ & $20.34(1.38,39.31)$ \\
\hline Libya (2015) & $\longrightarrow$ & $34.96(16.47,53.44)$ \\
\hline Tanzania (2015) & $\longrightarrow$ & $29.67(17.00,42.34)$ \\
\hline Algeria (2016) & $\longrightarrow$ & $26.57(15.37,37.77)$ \\
\hline Morocco (2016) & $\longrightarrow$ & $23.11(14.89,31.32)$ \\
\hline Algeria (2016) & $\longrightarrow$ & $23.04(15.29,30.79)$ \\
\hline Uganda (2016) & $\longrightarrow$ & $22.87(15.51,30.22)$ \\
\hline Egypt (2017) & $\longrightarrow$ & $20.93(14.78,27.08)$ \\
\hline Egypt (2017) & $\multimap$ & $20.58(14.74,26.42)$ \\
\hline Egypt (2017) & $\multimap$ & $23.44(17.74,29.13)$ \\
\hline Egypt (2018) & $\rightarrow-$ & $22.60(17.26,27.94)$ \\
\hline Nigeria (2018) & $\rightarrow$ & $21.03(16.64,25.43)$ \\
\hline Sudan (2018) & $\rightarrow-$ & $22.52(17.99,27.06)$ \\
\hline Egypt (2018) & $\bullet-$ & $24.20(19.55,28.85)$ \\
\hline Egypt (2018) & $\rightarrow$ & $27.76(22.89,32.62)$ \\
\hline Uganda (2019) & $\bullet$ & $26.10(22.59,29.60)$ \\
\hline Egypt (2019) & $\bullet$ & $25.65(22.16,29.14)$ \\
\hline Tunisia (2019) & $\rightarrow$ & $25.42(21.97,28.87)$ \\
\hline Egypt (2019) & $\rightarrow$ & $24.74(21.40,28.08)$ \\
\hline Algeria (2019) & $\rightarrow$ & $24.24(20.97,27.51)$ \\
\hline 1 & \multirow{2}{*}{\multicolumn{2}{|c|}{$\begin{array}{c}1 \\
60.8\end{array}$}} \\
\hline-60.8 & & \\
\hline
\end{tabular}

FIgURE 7: Cumulative meta-analysis of CPPA between 2014 and 2019 G.C. in Africa.

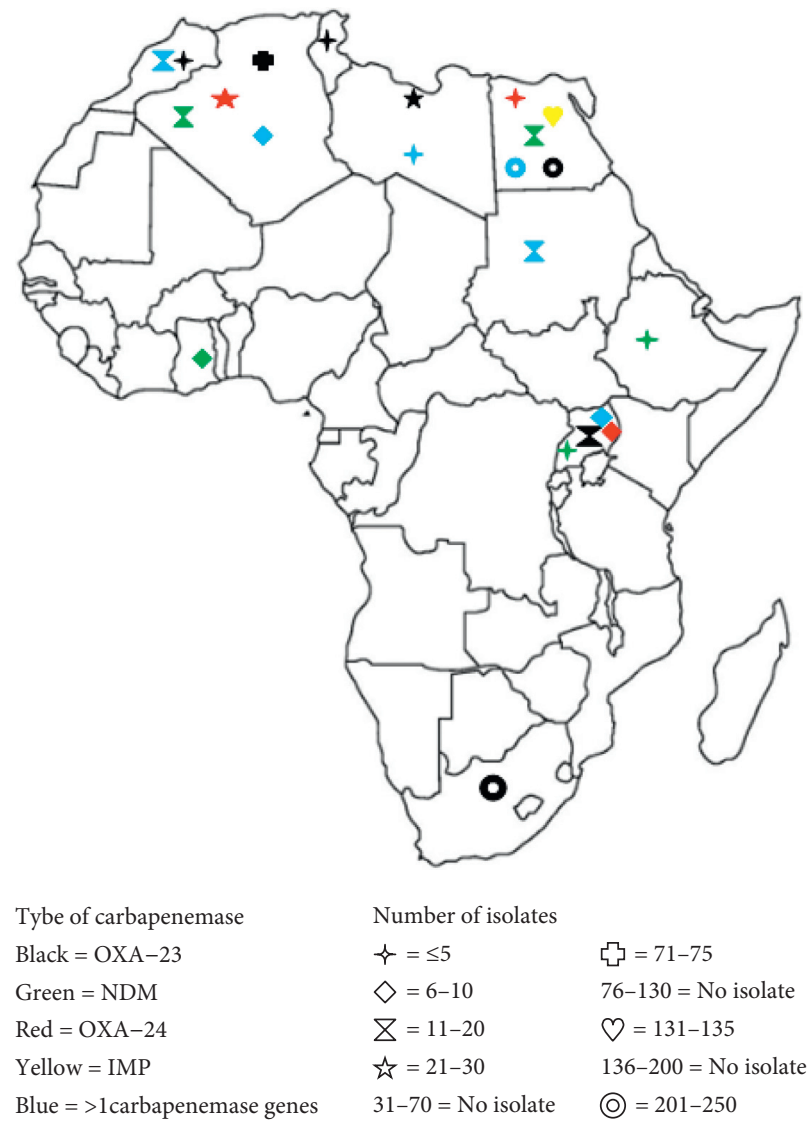

Figure 8: Geographical distribution of CPAB isolates in Africa from 2007 to 2017 G.C. 


\begin{tabular}{|c|c|c|c|c|}
\hline \multicolumn{2}{|l|}{$\begin{array}{l}\text { Study } \\
\text { ID }\end{array}$} & \multicolumn{2}{|r|}{ ES $(95 \% \mathrm{CI})$} & \multirow{2}{*}{$\begin{array}{c}\% \\
\text { weight } \\
7.25\end{array}$} \\
\hline Algeria (2018) & $\longrightarrow 1$ & - & $50.00(21.71,78.29)$ & \\
\hline Egypt (2017) & & - & $95.00(92.45,97.55)$ & 7.83 \\
\hline Egypt (2015) & & $\rightarrow$ & $80.67(74.35,86.99)$ & 7.80 \\
\hline Egypt (2017) & $\longrightarrow$ & & $39.53(24.92,54.15)$ & 7.67 \\
\hline Egypt (2018) & & & $60.00(46.42,73.58)$ & 7.69 \\
\hline Egypt (2019) & & $\rightarrow$ & $89.83(82.12,97.54)$ & 7.79 \\
\hline Libya (2015) & & $\longrightarrow$ & $88.00(75.26,100.74)$ & 7.71 \\
\hline Morocco (2019) & $\rightarrow-$ & & $24.69(15.30,34.08)$ & 7.76 \\
\hline Ethiopia (2017) & $\longrightarrow$ & & $23.08(0.17,45.98)$ & 7.44 \\
\hline Uganda (2016) & $\longrightarrow$ & & $24.14(8.56,39.71)$ & 7.65 \\
\hline Uganda (2019) & - & & $4.69(2.73,6.64)$ & 7.83 \\
\hline South Africa (2018) & & $\star$ & $96.45(93.40,99.51)$ & 7.82 \\
\hline South Africa (2015) & & & $61.86(52.19,71.52)$ & 7.76 \\
\hline Algeria (2016) & & & (Excluded) & 0.00 \\
\hline Overall $(I$-squared $=99.7 \%, p=0.000)$ & & & $56.97(28.92,85.02)$ & 100.00 \\
\hline \multicolumn{5}{|l|}{ NOTE: weights are from random-effects analysis } \\
\hline $\begin{array}{c}1 \\
-101\end{array}$ & 0 & 10 & & \\
\hline
\end{tabular}

FIGURE 9: Forest plot for the pooled prevalence of CPAB isolates in Africa from 2007 to 2017 G.C.

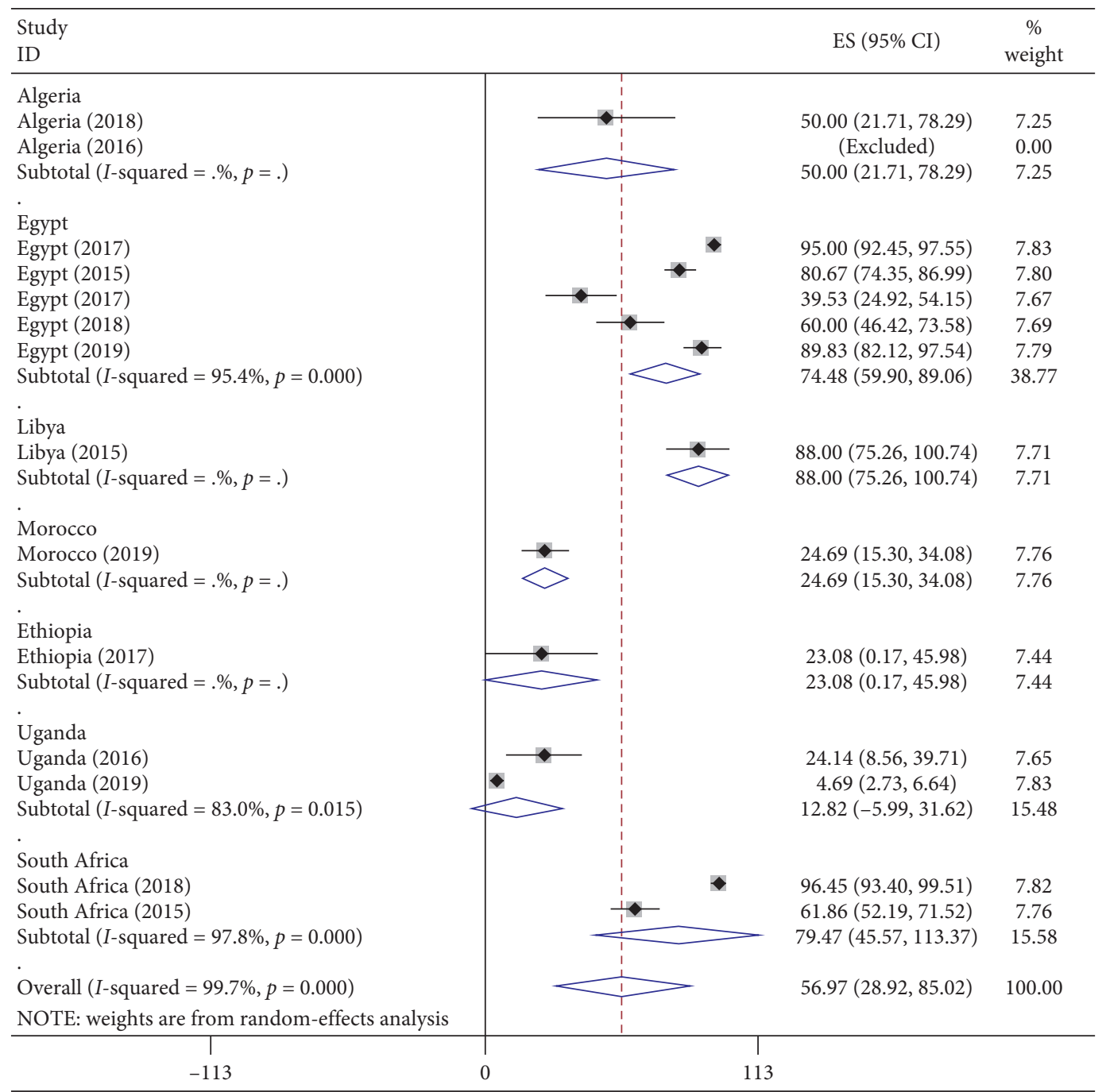

FIGURE 10: Subgroup analysis for the pooled prevalence of CPAB in Africa by country from 2007 to 2017 G.C. 


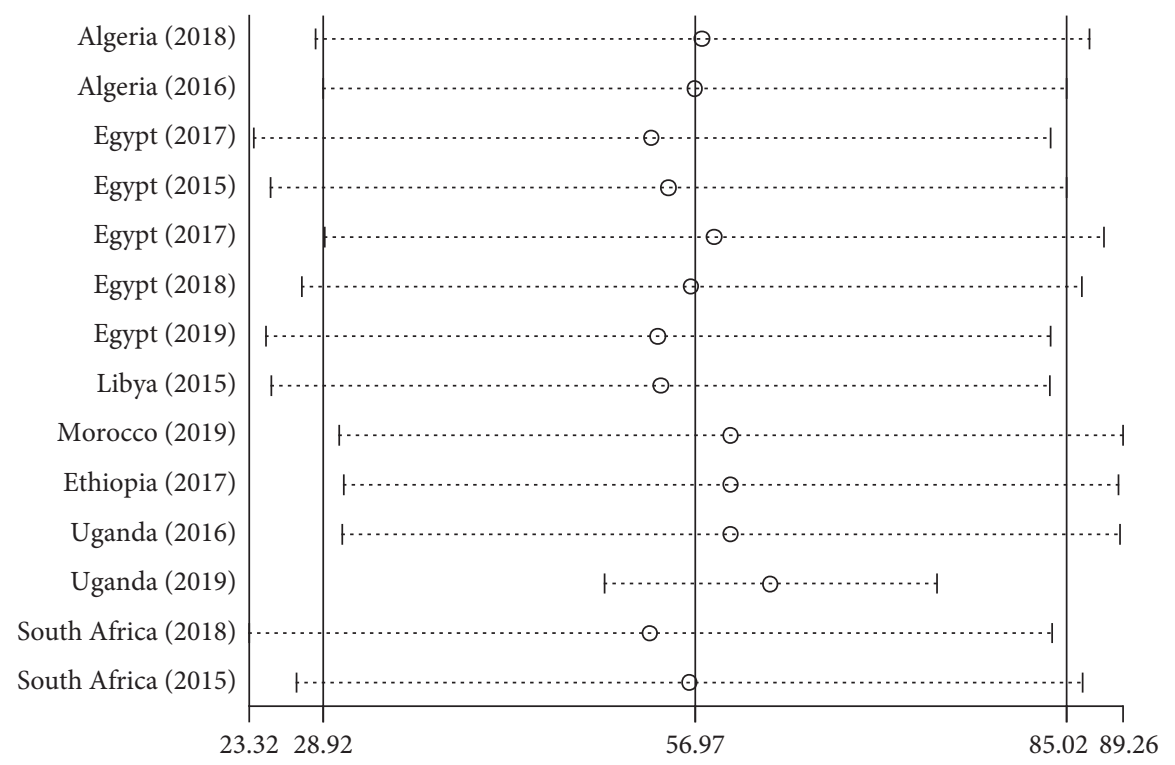

Meta-analysis estimates, given named study is omitted
| Lower CI limit
Estimate
| Upper CI limit

FIgURE 11: Sensitivity analysis for the pooled prevalence of CPAB in Africa from 2007 to 2017 G.C.

specimens in Africa. It also showed the production of different carbapenemase enzymes or detected genes encoding for these enzymes. According to the eligibility criteria of this review, most of the studies of these isolates were conducted in the North Africa region, frequently in Egypt and Algeria. This is consistent with a previous systematic review study among bacterial species producing carbapenemase enzymes in Africa [60].

Although the number of studies varied based on the type of isolates, some of the studies were also done in West, East, and South Africa. But there was no report from Central Africa. This might show that the lack of data on antibiotic resistance in Africa is still a problem for developing and applying evidence-based infection control and prevention measures. Moreover, it might impede patient care and public health, leaving the population vulnerable.

The individual studies included in this systematic review and meta-analysis showed a wide variation on the prevalence of $\mathrm{CP}$ isolates in Africa, depending on the bacterial species and geographical region. Moreover, the review also revealed that the CP isolates were more distributed in North African countries. These differences could result from or be attributable to irregular and varied use of antibiotics, low quality of personal hygiene, and inadequate environmental cleaning and infection control policies.

The current review also showed that the pooled prevalence of CPPA was $21.96 \%$. According to this review, VIM carbapenemases were highly prevalent carbapenemase type among $P$. aeruginosa isolates in Africa. This is the same with systematic review and meta-analysis on MBL $P$. aeruginosa conducted in Iran [61]. Moreover, VIMproducing isolates of $P$. aeruginosa had been reported worldwide, including European countries [62] and some countries of Asia [63, 64]. In this review, VIM carbapenemases were widely distributed in most African regions such as in North, East, and West African countries. All types of carbapenemases among $P$. aeruginosa isolates were found only in Egypt.

According to the findings of this meta-analysis, the higher pooled prevalence of CPAB was observed in Africa. This showed that carbapenemase production among A. baumannii infection developed fast in African countries. This is also consistent with reviews that showed the fast increasing trend of carbapenem resistance among clinical A. baumannii isolates worldwide $[65,66]$. This might lead to a "post-antibiotic era" for A. baumannii infection in the near future if an immediate action is not taken by the responsible bodies.

The first case of the OXA-type enzyme was reported from a clinical A. baumannii isolate detected in Scotland in 1985. It was initially named ARI-1 (Acinetobacter resistant to imipenem) [67] and renamed OXA-23 after sequencing [68]. According to this review, the OXA-23 was the predominant carbapenemases in the African A. baumannii isolates. This is not surprising, since OXA-23 has been found around the world [69-75]. Importantly, in Africa (particularly in North Africa), studies showed that blaOXA-23 is associated with ISAba1 elements $[13,31,76]$ and predominantly belongs to clone ST2 [22, 23, 31, 76-78], which may enable rapid transmission among OXA-23-producing A. baumannii clinical isolates.

Metallo-beta-lactamase (MBL) was detected as a commonly prevailing carbapenemase in Africa among CPPA and $\mathrm{CPAB}$ bacterial isolates. This is consistent with other 


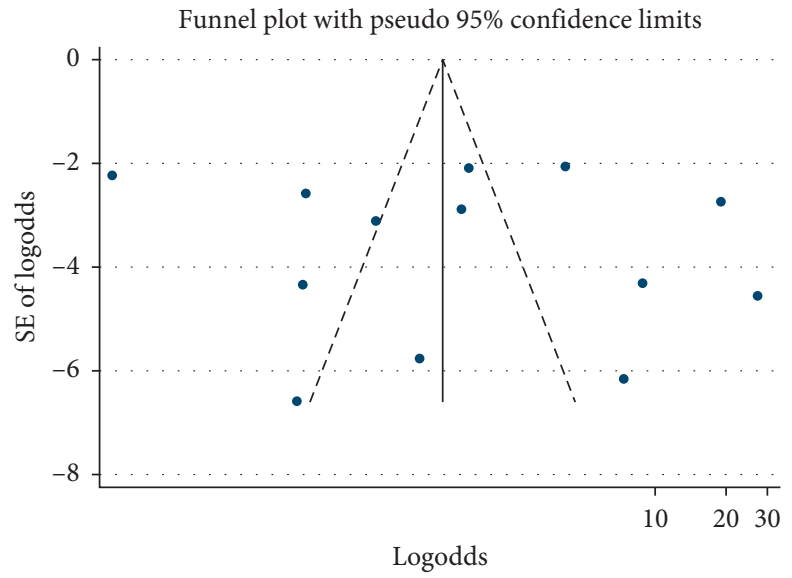

Figure 12: Funnel plot for publication bias of pooled prevalence of CPAB in Africa from 2007 to 2017 G.C.

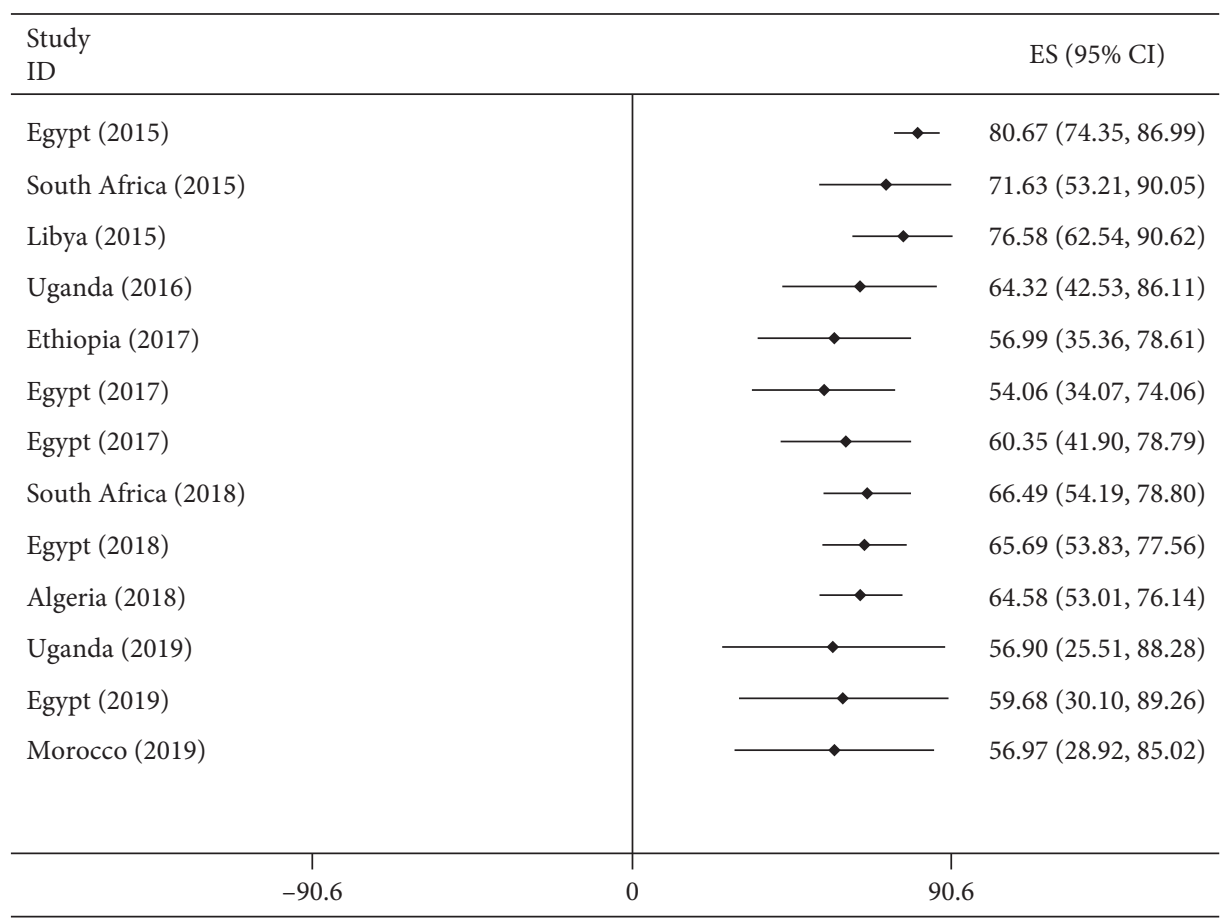

Figure 13: Cumulative meta-analysis of CPAB between 2015 and 2019 in Africa.

reviews that were conducted globally $[79,80]$. MBL genes are usually located in transferable genetic elements such as plasmid and integrons along with other antibiotic resistance genes. Therefore, dissemination of strains harboring MBL genes is of crucial importance, and appropriate measures should be taken into consideration by infection control programs [81].

The current review also showed the emergence of isolates coharboring genes encoding more than one class of carbapenemases in Northern, Eastern, and Western parts of African regions, but mostly in the North Africa region. Concomitant of blaOXA-23 + blaNDM frequently among A. baumannii clinical isolates was observed, particularly in Algeria, [28], Egypt [31, 33, 40], Morocco [45], and Uganda
[51]. Moreover, $P$. aeruginosa isolates coharboring genes encoding more than one carbapenemase were seen in different studies conducted in Egypt [11, 33, 34, 37, 41-43] and Sudan, [48, 49]. The emergence of such resistant strains represents a significant threat, not only to these countries but also to the whole of Africa and then to the world, especially as the dissemination of resistance genes is hastened by high rates of immigration and tourism [82-84].

Lastly, the time trend analysis for each of the bacterial isolates included in this review also showed that the prevalence of CPPA relatively increased over time in Africa. However, the prevalence of CPAB clinical isolates showed higher prevalence across the years in Africa. 
This review has some limitations: the first was considerable heterogeneity of studies included for the estimation of pooled prevalence of CPPA and CPAB. Therefore, to limit the influence of the study heterogeneity, the random-effects model of DerSimonian and Laird [85] was implemented in the meta-analyses, and subgroup analyses were also performed. Second, studies with different target populations, for example, neonates, adults, or transplant patients, were selected which might have an effect on the study. Third, since data for most parts of the region were not available, these findings might not represent the whole of Africa.

\section{Conclusion and Recommendations}

This review showed the pooled estimate prevalence of CPPA and $\mathrm{CPAB}$ across the African continent. It also revealed a high pooled prevalence of $\mathrm{CPAB}$ clinical isolates in Africa which needs an urgent action by the responsible bodies such as WHO and $\mathrm{FMOH}$, before the problem becomes totally uncontrollable. Moreover, the emergence of concomitant carbapenemase, OXA-23 + NDM, is in an alarming condition that needs further control and studies.

\section{Conflicts of Interest}

The authors declare that they have no conflicts of interest.

\section{Supplementary Materials}

Figure S1: forest plot for the pooled prevalence of CPPA among CRPA isolates in Africa. Figure S2: the subgroup analysis showed the pooled prevalence of CPPA isolates in Africa by region. Table S3: Egger's regression test for publication bias of CPPA. Figure S4: forest plot for the pooled prevalence of CPAB among CRAB isolates in Africa. Figure S5: the subgroup analysis showed the pooled prevalence of $\mathrm{CPAB}$ isolates in Africa by Region. Table S6: Egger's regression test for publication bias of CPAB. (Supplementary Materials)

\section{References}

[1] E. Tacconelli, E. Carrara, A. Savoldi, S. Harbarth, M. Mendelson, and D. L. Monnet, "Discovery, research, and development of new antibiotics: the WHO priority list of antibiotic-resistant bacteria and tuberculosis," The Lancet Infectious Diseases, vol. 18, no. 3, pp. 318-327, 2018.

[2] World Health Organization (WHO), Antimicrobial Resistance: Global Report on Surveillance, World Health Organization, Geneva, Switzerland, 2014.

[3] S. H. MacVane, "Antimicrobial resistance in the intensive care unit," Journal of Intensive Care Medicine, vol. 32, no. 1, pp. 25-37, 2017.

[4] F. Codjoe and E. Donkor, "Carbapenem resistance: a review," Medical Sciences, vol. 6, no. 1, 2017.

[5] J. M. Sahuquillo-Arce, A. Hernández-Cabezas, F. Yarad-Auad et al., "Carbapenemases: a worldwide threat to antimicrobial therapy," World Journal of Pharmacology, vol. 4, no. 1, pp. 75-95, 2015.
[6] R. P. Ambler, "The structure of beta-lactamases," Philosophical Transactions of the Royal Society of London. Series B, Biological Sciences, vol. 289, no. 1036, pp. 321-331, 1980.

[7] B. A. Evans and S. G. B. Amyes, "OXA -Lactamases," Clinical Microbiology Reviews, vol. 27, no. 2, pp. 241-263, 2014.

[8] S. Dahiya, P. Singla, U. Chaudhary, and B. Singh, "Carbapenemases: a review," International Journal of Advances in Health Sciences, vol. 2, no. 4, pp. 11-17, 2015.

[9] B. Bedenić and S. Sardelić, Carbapenemases. Growing and Handling of Bacterial Cultures, IntechOpen, London, UK, 2018.

[10] I. Maroui, A. Barguigua, A. Aboulkacem et al., "First report of VIM-2 metallo- $\beta$-lactamases producing Pseudomonas aeruginosa isolates in Morocco," Journal of Infection and Chemotherapy, vol. 22, no. 3, pp. 127-132, 2016.

[11] M. M. Zafer, M. H. Al-Agamy, H. A. El-Mahallawy, M. A. Amin, and S. E. D. Ashour, "Dissemination of VIM-2 producing Pseudomonas aeruginosa ST233 at tertiary care hospitals in Egypt," BMC Infectious Diseases, vol. 15, no. 1, p. 122, 2015.

[12] M. Wassef, M. Abdelhaleim, D. Ghaith, and Y. El-Mahdy, "Emerging New Delhi metallo- $\beta$-lactamase-1-type-producing gram-negative bacteria isolated from cairo university pediatric hospital, Cairo, Egypt," Journal of Global Antimicrobial Resistance, vol. 7, pp. 84-87, 2016.

[13] K. Khorsi, Y. Messai, M. Hamidi, H. Ammari, and R. Bakour, "High prevalence of multidrug-resistance in Acinetobacter baumannii and dissemination of carbapenemase-encoding genes blaOXA-23-like, blaOXA-24-like and blaNDM-1 in Algiers Hospitals," Asian Pacific Journal of Tropical Medicine, vol. 8, no. 6, pp. 438-446, 2015.

[14] A. Liberati, D. G. Altman, J. Tetzlaff et al., "The PRISMA statement for reporting systematic reviews and meta-analyses of studies that evaluate health care interventions: explanation and elaboration," PLoS Medicine, vol. 6, no. 7, 2009.

[15] M. Peters, C. Godfrey, P. McInerney, C. Soares, H. Khalil, and D. Parker, The Joanna Briggs Institute Reviewers' Manual 2015: Methodology for JBI Scoping Reviews, The Joanna Briggs Institute, Adelaide, Australia, 2015.

[16] J. P. A. Ioannidis, "Interpretation of tests of heterogeneity and bias in meta-analysis," Journal of Evaluation in Clinical Practice, vol. 14, no. 5, pp. 951-957, 2008.

[17] J. P. T. Higgins and S. G. Thompson, "Quantifying heterogeneity in a meta-analysis," Statistics in Medicine, vol. 21, no. 11, pp. 1539-1558, 2002.

[18] R. DerSimonian and N. Laird, "Meta-analysis in clinical trials," Controlled Clinical Trials, vol. 7, no. 3, pp. 177-188, 1986.

[19] H. Cooper, L. V. Hedges, and J. C. Valentine, The Handbook of Research Synthesis and Meta-Analysis, Russell Sage Foundation, New York, NY, USA, 2009.

[20] J. A. C. Sterne and M. Egger, "Funnel plots for detecting bias in meta-analysis," Journal of Clinical Epidemiology, vol. 54, no. 10, pp. 1046-1055, 2001.

[21] S. Duval and R. Tweedie, "A nonparametric "trim and fill" method of accounting for publication bias in meta-analysis," Journal of the American Statistical Association, vol. 95, no. 449 , pp. 89-98, 2000.

[22] N. Bourafa, W. Chaalal, S. Bakour et al., "Molecular characterization of carbapenem-resistant Gram-negative bacilli clinical isolates in Algeria," Infection and Drug Resistance, vol. 11, p. 735, 2018.

[23] F. Z. Mellouk, S. Bakour, S. Meradji et al., "First detection of VIM-4-producing Pseudomonas aeruginosa and OXA-48- 
producing Klebsiella pneumoniae in northeastern (annaba, skikda) Algeria," Microbial Drug Resistance, vol. 23, no. 3, pp. 335-344, 2017.

[24] S. Meradji, A. Barguigua, K. Zerouali et al., "Epidemiology of carbapenem non-susceptible Pseudomonas aeruginosa isolates in Eastern Algeria," Antimicrobial Resistance and Infection Control, vol. 4, 2015.

[25] S. Meradji, A. Barguigua, M. c. Bentakouk et al., "Epidemiology and virulence of VIM-4 metallo-beta-lactamase-producing Pseudomonas aeruginosa isolated from burn patients in Eastern Algeria,” Burns, vol. 42, no. 4, pp. 906-918, 2016.

[26] I. Sefraoui, M. Berrazeg, M. Drissi, and J.-M. Rolain, "Molecular epidemiology of carbapenem-resistant Pseudomonas aeruginosa clinical strains isolated from Western Algeria between 2009 and 2012," Microbial Drug Resistance, vol. 20, no. 2, pp. 156-161, 2014.

[27] M. Merradi, A. Kassah-Laouar, A. Ayachi et al., "Occurrence of VIM-4 metallo- $\beta$-lactamase-producing Pseudomonas aeruginosa in an Algerian hospital," The Journal of Infection in Developing Countries, vol. 13, no. 04, pp. 284-290, 2019.

[28] S. Bakour, A. Touati, T. Bachiri et al., "First report of $16 \mathrm{~S}$ rRNA methylase ArmA-producing Acinetobacter baumannii and rapid spread of metallo- $\beta$-lactamase NDM- 1 in Algerian hospitals," Journal of Infection and Chemotherapy, vol. 20, no. 11, pp. 696-701, 2014.

[29] M. R. Raouf, M. Sayed, H. A. Rizk, and N. A. Hassuna, "High incidence of MBL-mediated imipenem resistance among Pseudomonas aeruginosa from surgical site infections in Egypt," The Journal of Infection in Developing Countries, vol. 12 , no. 07 , pp. $520-525,2018$.

[30] N. M. Alkasaby and M. El Sayed Zaki, "Molecular study of Acinetobacter baumannii isolates for metallo- $\beta$-lactamases and extended-spectrum- $\beta$-lactamases genes in intensive care unit, mansoura university hospital, Egypt," International Journal of Microbiology, vol. 2017, Article ID 3925868, 6 pages, 2017.

[31] M. A. E.-G. El-Sayed, M. A. Amin, W. M. Tawakol, L. Loucif, S. Bakour, and J.-M. Rolain, "High prevalence of blaNDM-1 carbapenemase-encoding gene and 16S rRNA armA methyltransferase gene among Acinetobacter baumannii clinical isolates in Egypt," Antimicrobial Agents and Chemotherapy, vol. 59, no. 6, pp. 3602-3605, 2015.

[32] M. Assem, M. Abdalla Wifi, R. Elsherif, A. Saad, D. K. Ismail, and A. Hasanin, "Emergence of gram-negative bacilli with concomitant," American Journal of Internal Medicine, vol. 5, pp. 1-6, 2017.

[33] R. A. Ramadan, M. G. Gebriel, H. M. Kadry, and A. Mosallem, "Carbapenem-resistant Acinetobacter baumannii and Pseudomonas aeruginosa: characterization of carbapenemase genes and E-test evaluation of colistin-based combinations," Infection and Drug Resistance, vol. 11, 2018.

[34] H. O. Khalifa, A. M. Soliman, A. M. Ahmed et al., "High carbapenem resistance in clinical gram-negative pathogens isolated in Egypt," Microbial Drug Resistance, vol. 23, no. 7, pp. 838-844, 2017.

[35] A. F. Abaza, S. A. El Shazly, H. S. Selim, and G. S. Aly, "Metallo-beta-lactamase producing Pseudomonas aeruginosa in a healthcare setting in alexandria, Egypt," Polish Journal of Microbiology, vol. 66, no. 3, pp. 297-308, 2017.

[36] M. M. Zafer, M. H. Al-Agamy, H. A. El-Mahallawy, M. A. Amin, and M. S. E.-D. Ashour, "Antimicrobial resistance pattern and their beta-lactamase encoding genes among Pseudomonas aeruginosa strains isolated from cancer patients," BioMed Research International, vol. 2014, Article ID 101635, 8 pages, 2014.
[37] A. Gaballah, A. Elbaradei, A. Elsheredy, and O. Kader, "Emergence of bla VEB and bla GES among VIM-producing Pseudomonas aeruginosa clinical isolates in Alexandria, Egypt," Acta Microbiologica et Immunologica Hungarica, vol. 66, no. 1, pp. 131-142, 2018.

[38] M. H. Al-Agamy, N. G. Khalaf, M. M. Tawfick, A. M. Shibl, and A. El Kholy, "Molecular characterization of carbapeneminsensitive Acinetobacter baumannii in Egypt," International Journal of Infectious Diseases, vol. 22, pp. 49-54, 2014.

[39] D. M. Ghaith, M. M. Zafer, M. H. Al-Agamy, E. J. Alyamani, R. Y. Booq, and O. Almoazzamy, "The emergence of a novel sequence type of MDR Acinetobacter baumannii from the intensive care unit of an Egyptian Tertiary Care Hospital," Annals of Clinical Microbiology and Antimicrobials, vol. 16, no. 1, p. 34, 2017.

[40] L. Al-Hassan, M. M. Zafer, and H. El-Mahallawy, "Multiple sequence types responsible for healthcare-associated Acinetobacter baumannii dissemination in a single centre in Egypt," BioMed Central Infectious Diseases, vol. 19, no. 1, p. 829, 2019.

[41] N. M. Abdeltwab, M. Emara, and T. S. El-Mahdy, "First report of imipenem-resistant Pseudomonas aeruginosa clinical isolates harboring blaGIM-gene in the Middle East Region," Microbiology, vol. 52, 2019.

[42] S. M. Farhan, R. A. Ibrahim, K. M. Mahran, H. F. Hetta, and R. M. A. El-Baky, "Antimicrobial resistance pattern and molecular genetic distribution of metallo- $\beta$-lactamases producing Pseudomonas aeruginosa isolated from Hospitals in Minia, Egypt," Infection and Drug Resistance, vol. 12, p. 2125, 2019.

[43] H. Hashem, A. Hanora, S. Abdalla, A. Shaeky, and A. Saad, "Dissemination of metallo-beta-lactamase in Pseudomonas aeruginosa isolates in Egypt: mutation in blaVIM-4," APMIS: Acta Pathologica, Microbiologica, et Immunologica Scandinavica, vol. 125, no. 5, pp. 499-505, 2017.

[44] N. Mathlouthi, Z. Areig, C. Al Bayssari et al., "Emergence of carbapenem-resistant Pseudomonas aeruginosa and Acinetobacter baumannii clinical isolates collected from some Libyan hospitals," Microbial Drug Resistance, vol. 21, no. 3, pp. 335-341, 2015.

[45] H. El Hafa, K. Nayme, N. El Hamzaoui et al., "Dissemination of carbapenem-resistant Acinetobacter baumannii strains carrying the blaGES, blaNDM and blaOXA23 in Morocco," Germs, vol. 9, no. 3, p. 133, 2019.

[46] O. Dziri, C. A. ALONSO, R. Dziri et al., "Metallo- $\beta$-lactamases and class D carbapenemases in South-East Tunisia: implication of mobile genetic elements in their dissemination," International Journal of Antimicrobial Agents, vol. 52, no. 6, pp. 871-877, 2018.

[47] S. Chairat, H. Ben Yahia, B. Rojo-Bezares, Y. Sáenz, C. Torres, and K. Ben Slama, "High prevalence of imipenem-resistant and metallo-beta-lactamase-producing Pseudomonas aeruginosa in the Burns Hospital in Tunisia: detection of a novel class 1 integron," Journal of Chemotherapy, vol. 31, no. 3, pp. 120-126, 2019.

[48] M. A. Adam and W. I. Elhag, "Prevalence of metallo- $\beta$-lactamase acquired genes among carbapenems susceptible and resistant Gram-negative clinical isolates using multiplex PCR, Khartoum hospitals, Khartoum Sudan," BioMed Central Infectious Diseases, vol. 18, no. 1, p. 668, 2018.

[49] S. B. Satir, A. I. Elkhalifa, M. A. Ali, El Hussein, I. M. Elkhidir, and K. A. Enan, "Detection of Carbepenem resistance genes among selected Gram Negative bacteria isolated from patients in-Khartoum State, Sudan," Clinical Microbiology: Open Access, vol. 5, no. 6, 2016. 
[50] M. Pritsch, A. Zeynudin, M. Messerer et al., "First report on bla NDM-1-producing Acinetobacter baumannii in three clinical isolates from Ethiopia," BioMed Central Infectious Diseases, vol. 17, no. 1, p. 180, 2017.

[51] D. P. Kateete, R. Nakanjako, J. Namugenyi, J. Erume, M. L. Joloba, and C. F. Najjuka, "Carbapenem resistant Pseudomonas aeruginosa and acinetobacter baumannii at mulago hospital in kampala, Uganda (2007-2009)," Springerplus, vol. 5, no. 1, p. 1308, 2016.

[52] D. Aruhomukama, C. F. Najjuka, H. Kajumbula et al., "blaVIM- and blaOXA-mediated carbapenem resistance among Acinetobacter baumannii and Pseudomonas aeruginosa isolates from the Mulago Hospital Intensive Care Unit in Kampala," Uganda, vol. 19, no. 1, p. 853, 2019.

[53] S. Moyo, B. Haldorsen, S. Aboud et al., "Identification of VIM2-producing Pseudomonas aeruginosa from Tanzania is associated with sequence types 244 and 640 and the location of blaVIM-2 in a TniC integron," Antimicrobial Agents and Chemotherapy, vol. 59, no. 1, pp. 682-685, 2015.

[54] F. S. Codjoe, "Detection and characterisation of carbapenemresistant Gram negative bacilli infections in Ghana," ProQuest, 2017.

[55] K. Zubair and K. Iregbu, "Resistance pattern and detection of metallo-beta-lactamase genes in clinical isolates of Pseudomonas aeruginosa in a Central Nigeria Tertiary Hospital," Nigerian Journal of Clinical Practice, vol. 21, no. 2, pp. 176$182,2018$.

[56] M. Lowe, M. M. Ehlers, F. Ismail et al., "Acinetobacter baumannii: epidemiological and beta-lactamase data from two tertiary academic hospitals in tshwane, South Africa," Frontiers in Microbiology, vol. 9, p. 1280, 2018.

[57] E. E. Agoba, U. Govinden, A. K. C. Peer, J. Osei Sekyere, and S. Y. Essack, "IS Abal regulated OXA-23 carbapenem resistance in Acinetobacter baumannii strains in durban, South Africa," Microbial Drug Resistance, vol. 24, no. 9, pp. 1289-1295, 2018.

[58] T. Pedersen, J. O. Sekyere, U. Govinden et al., "Spread of plasmid-encoded NDM-1 and GES-5 carbapenemases among extensively drug-resistant and pandrug-resistant clinical Enterobacteriaceae in Durban, South Africa," Antimicrobial Agents and Chemotherapy, vol. 62, no. 5, pp. e02178-17, 2018.

[59] C. B. Adjei, U. Govinden, K. Moodley, and S. Y. Essack, "Molecular characterisation of multidrug-resistant Pseudomonas aeruginosa from a private hospital in durban, South Africa," Southern African Journal of Infectious Diseases, vol. 33, no. 2, pp. 38-41, 2018.

[60] R. I. Manenzhe, H. J. Zar, M. P. Nicol, and M. Kaba, "The spread of carbapenemase-producing bacteria in Africa: a systematic review," Journal of Antimicrobial Chemotherapy, vol. 70, no. 1, pp. 23-40, 2014.

[61] H. Vaez, F. Khademi, A. Salehi-Abargouei, and A. Sahebkar, "Metallo-beta-Lactamase-producing Pseudomonas aeruginosa in Iran: a systematic review and meta-analysis," Le Infezioni in Medicina, vol. 26, no. 3, pp. 216-225, 2018.

[62] M. Castanheira, L. M. Deshpande, A. Costello, T. A. Davies, and R. N. Jones, "Epidemiology and carbapenem resistance mechanisms of carbapenem-non-susceptible Pseudomonas aeruginosa collected during 2009-11 in 14 European and Mediterranean countries," Journal of Antimicrobial Chemotherapy, vol. 69, no. 7, pp. 1804-1814, 2014.

[63] S. M. Liew, G. Rajasekaram, S. D. Puthucheary, and K. H. Chua, "Detection of VIM-2-, IMP-1-and NDM-1producing multidrug-resistant Pseudomonas aeruginosa in
Malaysia," Journal of Global Antimicrobial Resistance, vol. 13, pp. 271-273, 2018.

[64] K. Osawa, K. Shigemura, K. Kitagawa et al., "Molecular characteristics of carbapenem-resistant Pseudomonas aeruginosa isolated from urine in Hyogo, Japan," International Journal of Urology, vol. 26, no. 1, pp. 127-133, 2019.

[65] A. J. Brink, "Epidemiology of carbapenem-resistant Gramnegative infections globally," Current Opinion in Infectious Diseases, vol. 32, no. 6, pp. 609-616, 2019.

[66] R. Xie, X. D. Zhang, Q. Zhao, B. Peng, and J. Zheng, “Analysis of global prevalence of antibiotic resistance in Acinetobacter baumannii infections disclosed a faster increase in OECD countries," Emerging Microbes \& Infections, vol. 7, no. 1, p. 31, 2018.

[67] R. Paton, R. Miles, J. Hood, and S. Amyes, “Ari 1: $\beta$-lactamasemediated imipenem resistance in Acinetobacter baumannii," International Journal of Antimicrobial Agents, vol. 2, no. 2, pp. 81-87, 1993.

[68] H. M. Donald, W. Scaife, S. G. Amyes, and H.-K. Young, "Sequence analysis of ARI-1, a novel OXA $\beta$-lactamase, responsible for imipenem resistance in Acinetobacter baumannii 6B92," Antimicrobial Agents and Chemotherapy, vol. 44, no. 1, pp. 196-199, 2000.

[69] R. Beigverdi, A. Sattari-Maraji, M. Emaneini, and F. Jabalameli, "Status of carbapenem-resistant Acinetobacter baumannii harboring carbapenemase: first systematic review and meta-analysis from Iran," Infection, Genetics and Evolution: Journal of Molecular Epidemiology and Evolutionary Genetics in Infectious Diseases, vol. 73, pp. 433-443, 2019.

[70] P. Nordmann, T. Naas, and L. Poirel, "Global spread of carbapenemase-producing Enterobacteriaceae," Emerging Infectious Diseases, vol. 17, no. 10, p. 1791, 2011.

[71] A. Liakopoulos, V. Miriagou, E. A. Katsifas et al., "Identification of OXA-23-producing Acinetobacter baumannii in Greece, 2010 to 2011," Euro Surveillance: Bulletin Europeen sur les Maladies Transmissibles=European Communicable Disease Bulletin, vol. 17, no. 11, 2012.

[72] N.-z Ning, X. Liu, C.-m Bao et al., "Molecular epidemiology of bla OXA-23-producing carbapenem-resistant Acinetobacter baumannii in a single institution over a 65-month period in North China," BioMed Central Infectious Diseases, vol. 17, no. 1, 2017.

[73] E. Ad Oliveira, G. Rd Paula, P. J. J. Mondino, T. P. G. Chagas, S. S. Bd Mondino, and Mendonça-Souza, "High rate of detection of OXA-23-producing acinetobacter from two general hospitals in Brazil," Revista da Sociedade Brasileira de Medicina Tropical, vol. 52, 2019.

[74] C. H. Rodríguez, M. Nastro, and A. Famiglietti, "Carbapenemases in Acinetobacter baumannii. Review of their dissemination in Latin America," Revista Argentina de Microbiologia, vol. 50, no. 3, pp. 327-333, 2018.

[75] A. Al Atrouni, M. Hamze, T. Jisr et al., "Wide spread of OXA23-producing carbapenem-resistant Acinetobacter baumannii belonging to clonal complex II in different hospitals in Lebanon," International Journal of Infectious Diseases, vol. 52, pp. 29-36, 2016.

[76] A. B. Benmahmod, H. S. Said, and R. H. Ibrahim, "Prevalence and mechanisms of carbapenem resistance among Acinetobacter baumannii clinical isolates in Egypt," Microbial Drug Resistance, vol. 25, no. 4, pp. 480-488, 2019.

[77] N. Mathlouthi, Y. Ben lamine, R. Somai et al., "Incidence of OXA-23 and OXA-58 carbapenemases coexpressed in clinical isolates of Acinetobacter baumannii in Tunisia," Microbial Drug Resistance, vol. 24, no. 2, pp. 136-141, 2018. 
[78] N. Mathlouthi, A. A. El Salabi, M. B. Jomàa-Jemili et al., "Early detection of metallo- $\beta$-lactamase NDM-1-and OXA-23 carbapenemase-producing Acinetobacter baumannii in Libyan hospitals," International Journal of Antimicrobial Agents, vol. 48, no. 1, pp. 46-50, 2016.

[79] D. van Duin and Y. Doi, "The global epidemiology of carbapenemase-producing Enterobacteriaceae," Virulence, vol. 8, no. 4, pp. 460-469, 2017.

[80] L. Dortet, L. Poirel, and P. Nordmann, "Worldwide dissemination of the NDM-type carbapenemases in Gramnegative bacteria," BioMed Research International, vol. 2014, Article ID 249856, 12 pages, 2014.

[81] H. Vaez, S. Moghim, B. Nasr Esfahani, and H. Ghasemian Safaei, "Clonal relatedness among imipenem-resistant Pseudomonas aeruginosa isolated from ICU-hospitalized patients," Critical Care Research and Practice, vol. 2015, Article ID 983207, 5 pages, 2015.

[82] H. Solgi, F. Badmasti, C. G. Giske, S. Aghamohammad, and F. Shahcheraghi, "Molecular epidemiology of NDM-1-and OXA-48-producing Klebsiella pneumoniae in an Iranian hospital: clonal dissemination of ST11 and ST893," Journal of Antimicrobial Chemotherapy, vol. 73, no. 6, pp. 1517-1524, 2018.

[83] A. Shibl, M. Al-Agamy, Z. Memish, A. Senok, S. A. Khader, and A. Assiri, "The emergence of OXA-48-and NDM-1positive Klebsiella pneumoniae in riyadh, Saudi Arabia," International Journal of Infectious Diseases, vol. 17, no. 12, pp. e1130-e1133, 2013.

[84] A. Uskudar-Guclu, M. Guney, A. K. Sig, S. Kilic, and M. Baysallar, "Arising prevalence of OXA-48 producer Escherichia coli and OXA-48 with NDM co-producer Klebsiella pneumoniae strains," Revista Romana de Medicina de Laborator, vol. 27, no. 3, pp. 319-326, 2019.

[85] R. DerSimonian and N. Laird, "Meta-analysis in clinical trials revisited," Contemporary Clinical Trials, vol. 45, pp. 139-145, 2015. 